\title{
Increased tibial tubercle-trochlear groove and patellar height indicate a higher risk of recurrent patellar dislocation following medial reefing
}

\author{
Marc-Daniel Ahrend ${ }^{1,2}$ (D) $\cdot$ Tobias Eisenmann $^{1} \cdot$ Moritz Herbst $^{1} \cdot$ Boyko Gueorguiev $^{2} \cdot$ Gabriel Keller $^{3}$. \\ Florian Schmidutz ${ }^{1} \cdot$ Stefan Döbele ${ }^{1} \cdot$ Steffen Schröter ${ }^{4} \cdot$ Christoph Ihle $^{1}$
}

Received: 1 November 2020 / Accepted: 13 April 2021 / Published online: 25 May 2021

(c) The Author(s) 2021

\begin{abstract}
Purpose Identifying anatomical risk factors on recurrent dislocation after medial reefing is important for deciding surgical treatment. The present study aimed to retrospectively analyze the preoperative magnetic resonance imaging (MRI)-based parameters of patients treated with medial reefing and whether these parameters lead to a higher risk of recurrent dislocation. Methods Fifty-five patients (18.6 \pm 6.6 years) who underwent medial reefing after primary traumatic patellar dislocation (84\% with medial patellofemoral ligament [MPFL] rupture) were included. Patients were followed up for at least 24 months postoperatively ( $3.8 \pm 1.2$ years) to assess the incidence of recurrent patellar dislocation. In patients without recurrent dislocation, the Kujala and subjective IKDC scores were assessed. Moreover, the tibial tubercle-trochlear groove (TT-TG), sulcus angle, patellar tilt, patellar shift, and lateral trochlea index (LTI) were measured. The patellar height was measured using the Caton-Dechamps (CDI), Blackburne-Peel (BPI), and Insall-Salvati index (ISI). The cohort was subclassified into two groups with and without recurrent dislocation. Differences between groups were analyzed with respect to the MRI parameters.

Results Forty percent had a pathological sulcus angle of $>145^{\circ}, 7.2 \%$ had an LTI of $<11^{\circ}, 47.3 \%$ had a patellar tilt of $>20^{\circ}$, and $36.4 \%$ had a TT-TG of $\geq 16 \mathrm{~mm}$. Increased patellar height was observed in $34.5,65.5$, and $34.5 \%$ of the patients as per CDI, BPI, and ISI, respectively. Nineteen (34.5\%) patients suffered from recurrent dislocation. Compared with patients without recurrent dislocation, those with recurrent dislocation had a significantly lower LTI $(p=0.0467)$. All other parameters were not significantly different between the groups. Risk factor analysis showed higher odds ratios $(\mathrm{OR}>2)$, although not statistically significant, for MPFL rupture (OR 2.05 [95\% confidence interval 0.38-11.03], LTI (6.6 [0.6-68.1]), TT-TG (2.9 [0.9-9.2]), and patellar height according to ISI (2.3 [0.7-7.5]) and CDI (2.3 [0.7-7.5])). Patients without recurrent dislocation had a Kujala score of $93.7 \pm 12.1$ (42-100) points and an IKDC score of 90.6 \pm 11.7 (55.2-100) points.

Conclusion Anatomical, MRI-based parameters should be considered before indicating medial reefing. A ruptured MPFL, an LTI $<11^{\circ}$, a TT-TG $\geq 16 \mathrm{~mm}$, a patellar tilt $>20 \mathrm{~mm}$, and an increased patellar height according to ISI and CDI were found to be associated, although not significantly, with a higher risk $(\mathrm{OR}>2)$ of recurrent patellar dislocation after medial reefing. Thorough preoperative analysis is crucial to reduce the risk of recurrent dislocation in young patient cohorts.
\end{abstract}

Level of evidence Level IV

Keywords Patellar dislocation $\cdot$ Medial reefing $\cdot$ Recurrent dislocation $\cdot$ MRI

Moritz Herbst

mherbst2@bgu-tuebingen.de

1 Department of Traumatology and Reconstructive Surgery, BG Trauma Center Tübingen, Eberhard Karls University Tübingen, Tübingen, Germany

2 AO Research Institute Davos, Davos, Switzerland

3 Department of Diagnostic and Interventional Radiology, University Hospital Tuebingen, Tübingen, Germany

4 Department of Traumatology and Reconstructive Surgery, Diakonie Klinikum GmbH Jung-Stilling-Krankenhaus, Siegen, Germany

\section{Introduction}

Patellar dislocation is a common knee injury with a primary patellar dislocation incidence of up to $108 / 100,000$ in Western Europe between the age of 10 and 19 years [19, 45]. The first dislocation is often a result of a traumatic event and can lead to recurrent patellar dislocation with a reduced quality of life [27]. Recurrent patellar dislocation is reported in $22.7-29.4 \%$ depending on the injury pattern, various anatomical risk factors assessed by magnetic resonance imaging 
(MRI), or radiographs as well as the applied treatment option $[15,18,19]$.

Thus, there is no single gold standard or treatment algorithm available for treating primary patellar dislocation. Whether it can be treated surgically or non-surgically is still a subject of debate, and there is insufficient evidence to support either of the options $[40,44]$. According to the best available evidence, surgical treatment of acute patellar dislocation may result in a lower rate of recurrent dislocations than non-surgical treatment; however, functional outcome scores are not improved by surgical treatment [15, 35]. Surgical reconstruction of the medial patellofemoral ligament (MPFL) is widely performed, and there are several publications available that report different techniques, outcomes after treatment, and the analysis of the potential risk factors of recurrent dislocation [6, 16, 30, 31, 41]. By contrast, medial reefing is a less invasive procedure but also less evaluated. Moreover, good clinical outcome has been reported following this treatment [20]. Nevertheless, the indications and contraindications of medial reefing have been rarely described, and individual anatomy that predisposes one to primary or recurrent dislocation has not been comprehensively considered. In particular, anatomical parameters based on MRI scans before medial reefing have not been described yet. Moreover, an analysis of potential anatomical risk factors on recurrent dislocation after medial reefing is still lacking.

Therefore, this study primarily aimed to retrospectively analyze the preoperative MRI-based parameters of patients who were treated with medial reefing to provide further information on the indication of medial reefing. The secondary aim of this study was to analyze whether these MRIbased anatomical parameters influence the risk of recurrent dislocation after medial reefing. It was hypothesized that patients with trochlear dysplasia and increased patellar height were more likely to experience recurrent dislocation after medial reefing.

\section{Materials and methods}

The local ethics committee (195/2014BO2) reviewed and approved the study protocol, and informed consent was obtained from all included subjects. Between 2004 and 2013, 316 patients presented with primary patellar dislocation at a level-1 trauma center in Western Europe. Patients with primary patellar dislocation and initial medial reefing were included, whereas patients with missing data, including a missing MRI scan directly following primary patellar dislocation, or other treatments, such as non-surgical treatment, MPFL reconstruction, or an osteotomy, were excluded. Patients were followed up for at least 24 months postoperatively ( $3.8 \pm 1.2$ [range 2.1-7.5 years]) to assess the incidence of recurrent patellar dislocation following medial reefing. During the follow-up, the Kujala [13] and the subjective IKDC scores [23] were assessed in patients without recurrent dislocation to analyze functional outcome after medial reefing. The patient flow chart is presented in Fig. 1 .

\section{Arthroscopic medial reefing}

The indication for the surgical treatment of patellar dislocation was a traumatic patellar dislocation. Surgery was performed in an average of $38.7 \pm 50.3$ days following the primary dislocation. Surgery was performed as described by Ihle et al. [20]. A Vicryl CTX sized 0 suture (Johnson \& Johnson Medical GmbH, Ethicon Germany, Norderstedt, Germany) was placed at the proximal and medial borders of the patella directly next to the bone and taken out approximately $3 \mathrm{~cm}$ posteromedial. A small skin incision at the insertion of the suture was performed, and the suture was passed percutaneously via the capsule to the extra-articular area. Then, with the same technique, a second and a third suture were positioned $1.5 \mathrm{~cm}$ distally to the above suture. The knot was tightened after removing the intra-articular fluid at $30^{\circ}$ knee flexion.

Full weight-bearing was allowed immediately after surgery. The active and passive range of motion was limited between $0^{\circ}$ and $60^{\circ}$ for the first 3 weeks and $0^{\circ}$ and $90^{\circ}$ for additional 3 weeks; return-to-sport was allowed after 12 weeks.

\section{MRI evaluation}

All MRI scans were analyzed by a radiologist with respect to bone bruises, cartilage lesions, and ruptures of MPFL or the medial retinaculum. The following MRI-based radiological parameters were selected based on commonly cited literature $[2,37]$ and measured twice by two orthopedic surgeons independently at two different time points ( 2 weeks interval): tibial tubercle-trochlear groove (TT-TG) [38], sulcus angle [36], patellar tilt [14], patellar shift [37], lateral trochlea index (LTI) [10] (Fig. 2), and patellar height parameters (patellotrochlear index [PTI) according to Biedert and Albrecht [4], Koshino index, Caton-Dechamps index [CDI], Blackburne-Peel index [BPI], Insall-Salvati index [ISI]). The imaging software Osirix (Pixmeo, Geneva, Switzerland) was used for analysis. The observers were blinded to the incidence of recurrent dislocation. The measurement accuracy for distances $(\mathrm{mm})$ and angles was one decimal. Patellar height indices were expressed with two decimals.

TT-TG was measured on two axial slices according to Schoettle et al. [38] between the deepest cartilaginous point of the trochlear groove and the tibial tuberosity at the patellar insertion. A baseline was drawn tangent to the cartilaginous border of the posterior condyles and a 
Fig. 1 Patient flow chart with exclusion criteria

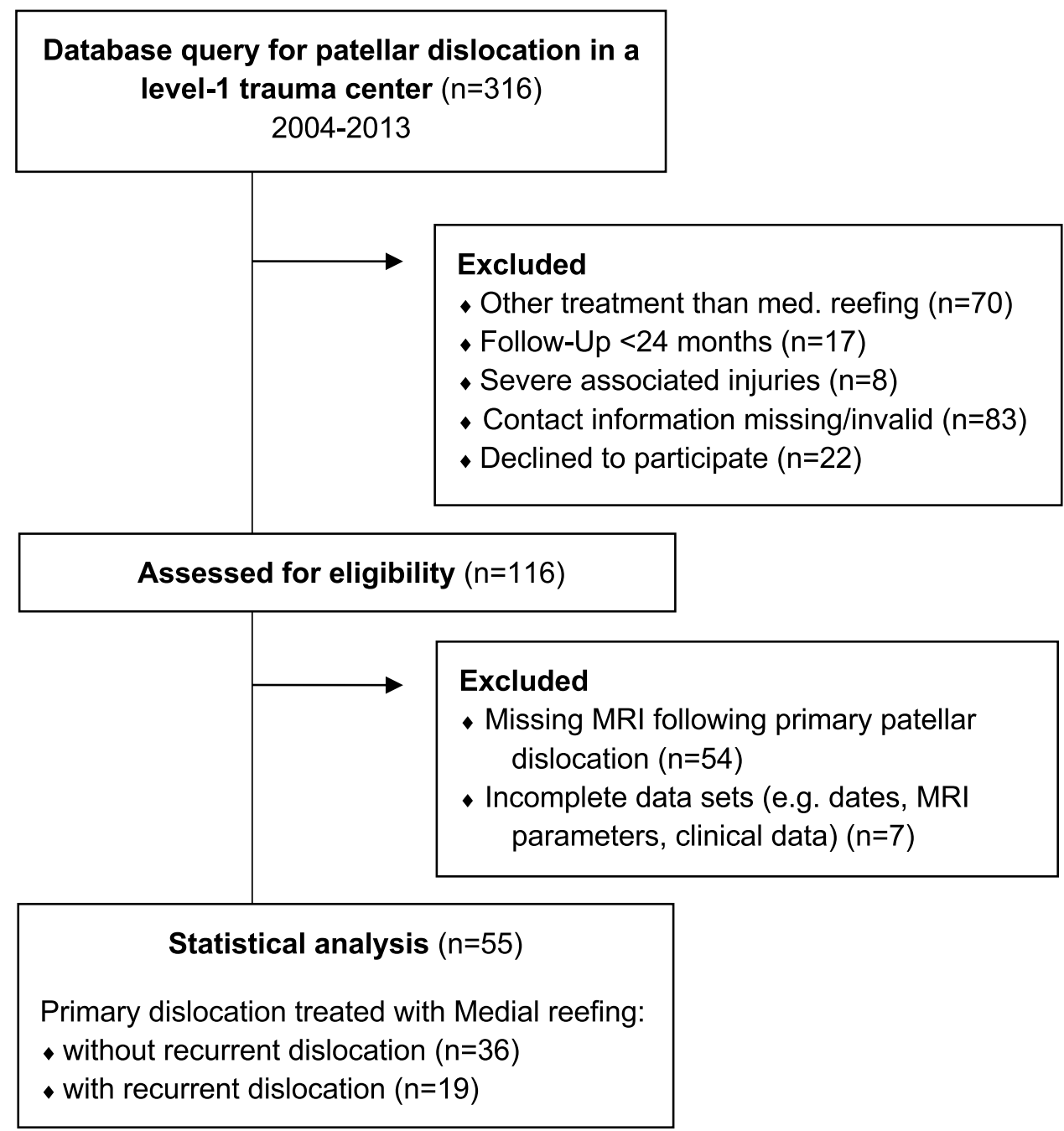

perpendicular line (B) to the deepest cartilaginous point of the trochlear groove. A second image was superimposed over the first image. The second image showed the attachment of the patellar tendon. A line $(\mathrm{C})$ was drawn parallel to the first line via the center of the patellar insertion. Then, the distance (A) between the perpendicular lines B and $\mathrm{C}$ was measured (TT-TG).

Patellar tilt was measured on an axial view as per Dejour et al. [14]. A baseline (B) was drawn tangent to the cartilaginous border of the posterior condyles. On a second image depicting the greatest patellar width, a line (A) was drawn from the medial to the lateral border of the patella. The angle between both lines was defined as the patellar tilt.

To measure patellar shift, the distance between the two parallel lines was measured. Line B was drawn via the deepest point of the trochlear groove perpendicular (A) to the posterior femoral condyle aspect. Line $\mathrm{C}$ was parallel to $\mathrm{B}$ and via the most posterior aspect of the retropatellar surface [37].
According to Carrillon et al. [10], LTI describes the morphology of the trochlear groove. It was determined by the angle between the posterior aspect of the femoral condyles (line B) and the cartilaginous surface of the lateral trochlea (A). Line A was drawn on the first craniocaudal axial view showing full articular cartilage over the lateral trochlear facet.

The sulcus angle of the trochlear groove was determined using the first transverse craniocaudal image that shows a complete cartilaginous trochlear surface. It describes the angle between the medial and the lateral aspect of the trochlear groove $[33,36]$.

Besides CDI [11], BPI [7], and ISI [22], PTI as per Biedert and Albrecht [4] was measured in sagittal slices. PTI is the overlap percentage of the patellar cartilage (line A) and the trochlear cartilage $(\mathrm{E})$. The trochlear cartilage is measured parallel to the patellar cartilage from the superior aspect of the trochlear cartilage to a reference line perpendicular to the inferior end of line A. The Koshino index was also measured in sagittal slices. Three lines were drawn, and the 

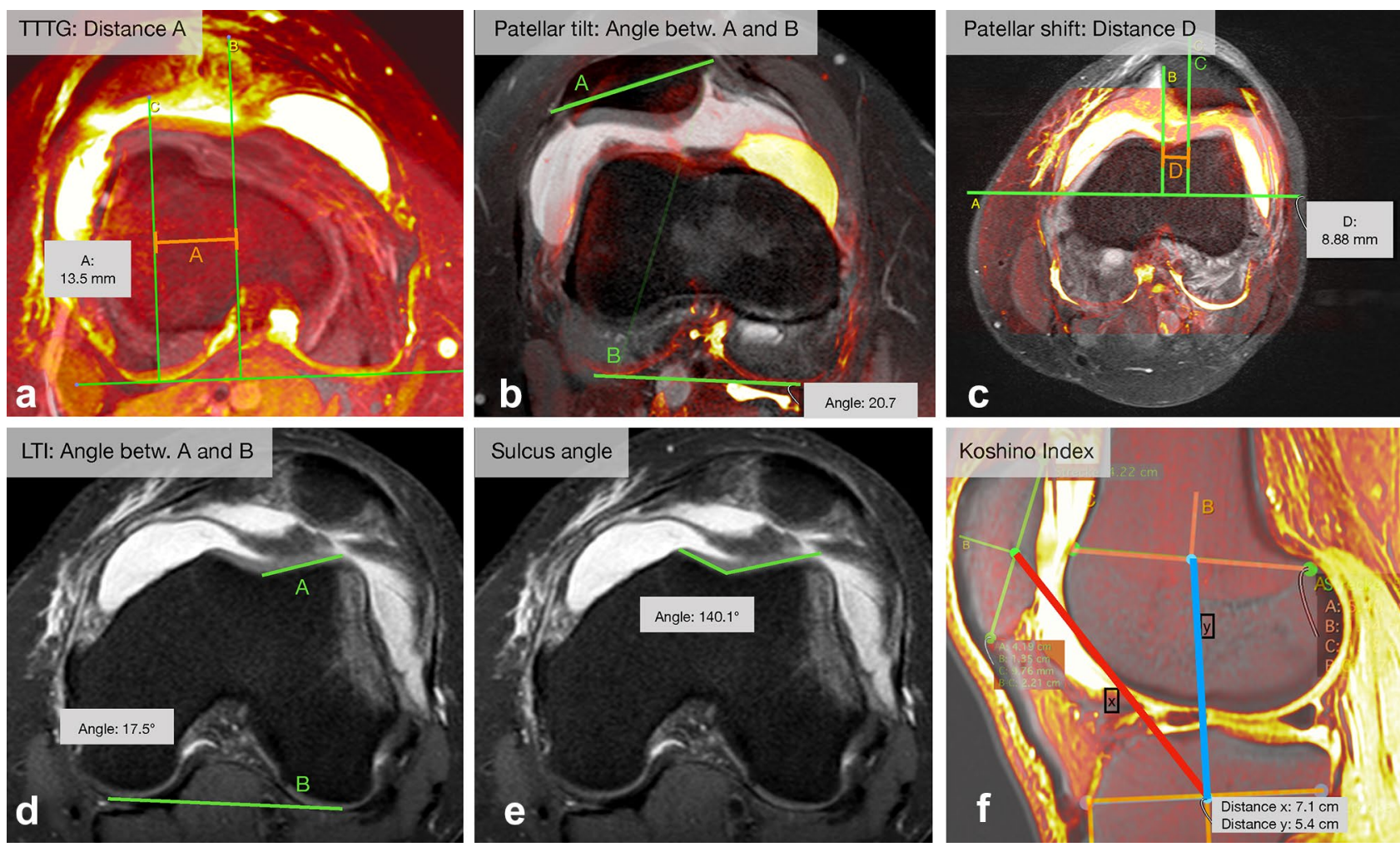

Fig. 2 Overview of MRI parameters measured with Osirix. a Overlapped axial slices to measure TT-TG (tibial tubercle-trochlear groove), b patellar tilt $\mathbf{c}$ patellar shift. $\mathbf{d}$ axial images to measure LTI (lateral trochlear index), e the sulcus angle. $\mathbf{f}$ sagittal image to measure the Koshino index

means of these parameters as well as demographic data were descriptively analyzed. Data are presented as absolute and relative values $(n(\%))$ as well as means \pm standard deviation (minimum-maximum). The cohort was subclassified into two groups: patients with and without recurrent dislocation. Data distribution was analyzed using the Shapiro-Wilk test, and groups were tested with respect to differences in demographic and MRI parameters. Independent $t$ tests were used for normally distributed data. The Mann-Whitney $U$ test was used when data were not normally distributed. Bivariate data were tested using the Chi-square test. For each MRI parameter, a threshold between the normal and abnormal value was defined based on literature recommendation (Table 1). The odds ratio (OR) of each factor was calculated using contingency tables.

The significance level was set at 5\%. Statistical analysis was performed using JMP® (SAS Institute Inc., JMP®, Version 13.0.0, Cary, NC, USA) and STATA $®$ (Stata Corporation, 15.0, College Station, TX, USA). 
Table 1 Distribution of preoperative MRI parameters in patients underwent medial reefing (mean $\pm \mathrm{SD}$ (minimummaximum) or $n(\%)$ )

\begin{tabular}{lllc}
\hline & Reference/normal values & $\begin{array}{l}\text { Number }(\%) \text { of patients } \\
\text { with abnormal values }\end{array}$ & Mean \pm SD (min.-max.) \\
\hline Sulcus Angle $\left(^{\circ}\right)$ & $\leq 145^{\circ}[47]$ & $22(40.0 \%)$ & $142.7 \pm 6.0(126.8-152.6)$ \\
Lateral trochlea index $\left(^{\circ}\right)$ & $>11^{\circ}[10,25]$ & $4(7.2 \%)$ & $17.6 \pm 5.0(7.1-32.4)$ \\
TT-TG $(\mathrm{mm})$ & $<16 \mathrm{~mm} \mathrm{[3]}$ & $20(36.4 \%)$ & $14.4 \pm 4.1(4.9-22.6)$ \\
Patella tilt $\left(^{\circ}\right)$ & $\leq 20^{\circ}[3,14]$ & $26(47.3 \%)$ & $20.2 \pm 7.0(7.8-41.7)$ \\
Patella shift $(\mathrm{mm})$ & $<2.5 \mathrm{~mm}[37]$ & $47(85.5 \%)$ & $7.2 \pm 4.4(-0.6-21.0)$ \\
Patellar height & & & $0.47 \pm 0.16(0.19-0.89)$ \\
Patellotrochlea index & $0.13-0.50[4]$ & 0 & $1.25 \pm 0.07(1.04-1.39)$ \\
Koshino index & $0.99-1.20[28]$ & $38(69.1 \%)$ & $1.23 \pm 0.13(0.97-1.51)$ \\
CDI & Patella alta: $>1.3[47]$ & $39(34.5 \%)$ & $1.06 \pm 0.15(0.80-1.34)$ \\
BPI & Patella alta: $>1.0[5]$ & $36(65.5 \%)$ & $1.20 \pm 0.18(0.78-1.56)$ \\
ISI & Patella alta: $>1.3[1]$ & $19(34.5 \%)$ & \\
\hline
\end{tabular}

\section{Results}

Fifty-five patients $(18.6 \pm 6.6$ years [range $10-43$ ], female: 23 [41.8\%], male: 32 [58.2\%]) were included in the study, and they underwent medial reefing after primary patellar dislocation.

MRI parameters are presented in Table 1; they showed moderate to excellent intra- and interobserver reliability (Table 2). Forty-four $(80.0 \%)$ patients had bone bruises at the femoral condyle, whereas 42 (76.4) had bone bruises at the patella A cartilage lesion at the patellar was identified in 31 patients $(56.4 \%)$ and at the femoral condyle in $22(40.0 \%)$ patients. MRI revealed an osteochondral flake in $14(25.5 \%)$ patients, an MPFL rupture in 46 (83.6\%), and a medial retinaculum rupture in 43 (78.2\%).

In the cohort, $40.0 \%$ of the patients had a pathological sulcus angle of $>145^{\circ}, 7.2 \%$ had a low LTI of $<11^{\circ}$, $47.3 \%$ had a patellar tilt of $>20^{\circ}$, and $36.4 \%$ had a TT-TG of $\geq 16 \mathrm{~mm}$. Increased patellar height was identified in
$34.5 \%$ of the patients according to CDI, $65.5 \%$ as per BPI, $34.5 \%$ according to ISI, $69.1 \%$ as per the Koshino index, and no patient had an increased patellar height according to PTI.

Nineteen $(34.5 \%)$ patients who underwent medial reefing experienced recurrent patellar dislocation. Patients with recurrent dislocation had a significantly lower LTI than those without recurrent dislocation $(p=0.0467)$. All other parameters did not differ significantly between patients with and without recurrent patellar dislocation (Table 3). Risk factor analysis (Table 4) revealed no significant risk factor in the cohort, which was expressed by the CI including 1 . Parameters with the largest ORs included LTI, TT-TG, and patellar height as per ISI and CDI. Moreover, patients with ruptured MPFL (OR 2.05 [95\% CI 0.38-11.03]) or ruptured medial reticulum (OR 1.78 [95\% CI 0.42-7.54]) were more likely to suffer from a recurrent dislocation. Patients without recurrent dislocation had a Kujala score of $93.7 \pm 12.1(42-100)$ and a subjective IKDC score of $90.6 \pm 11.7$ (55.2-100) points.

Table 2 Intra- and interobserver reliability (ICC (95\%CI)) and intra- and interobserver difference (mean \pm standard deviation (minimum-maximum))

\begin{tabular}{lllll}
\hline & Intraobserver reliability & $\begin{array}{l}\text { Intraobserver differences }- \\
\text { absolute values }\end{array}$ & Interobserver reliability & $\begin{array}{l}\text { Interobserver differences }- \\
\text { absolute values }\end{array}$ \\
\hline Sulcus Angle & $0.88(0.80-0.93)$ & $2.7 \pm 2.9(0-14.0)$ & $0.74(0.69-0.84)$ & $3.8 \pm 2.7(0.1-11.1)$ \\
LTI & $0.84(0.74-0.90)$ & $2.2 \pm 2.0(0-10.3)$ & $0.86(0.78-0.92)$ & $2.3 \pm 1.6(0.4-7.6)$ \\
TT-TG & $0.90(0.83-0.94)$ & $1.7 \pm 1.2(0.2-5.2)$ & $0.82(0.71-0.89)$ & $2.0 \pm 1.7(0-9.2)$ \\
Patella tilt & $0.93(0.88-0.96)$ & $2.7 \pm 2.2(0.2-12.7)$ & $0.92(0.87-0.95)$ & $2.3 \pm 2.1(0.1-10.4)$ \\
Patella shift & $0.94(0.89-0.96)$ & $1.3 \pm 1.1(0.1-5.1)$ & $0.89(0.82-0.94)$ & $1.7 \pm 1.3(0-7.1)$ \\
PTI & $0.81(0.70-0.89)$ & $0.05 \pm 0.08(0-0.56)$ & $0.81(0.69-0.88)$ & $0.06 \pm 0.09(0-0.64)$ \\
Koshino index & $0.79(0.70-0.87)$ & $0.04 \pm 0.04(0-0.22)$ & $0.52(0.29-0.69)$ & $0.04 \pm 0.08(0-0.57)$ \\
CDI & $0.72(0.56-0.83)$ & $0.08 \pm 0.07(0-0.39)$ & $0.85(0.76-0.91)$ & $0.07 \pm 0.05(0-0.23)$ \\
BPI & $0.78(0.64-0.86)$ & $0.08 \pm 0.09(0-0.39)$ & $0.76(0.62-0.85)$ & $0.08 \pm 0.08(0-0.42)$ \\
ISI & $0.89(0.81-0.93)$ & $0.06 \pm 0.10(0-0.34)$ & $0.94(0.90-0.96)$ & $0.05 \pm 0.04(0-0.20)$ \\
\hline
\end{tabular}


Table 3 Distribution of MRI parameters in patients with and without recurrent dislocation $($ mean $\pm \mathrm{SD}(95 \% \mathrm{CI}) ; n(\%))$
Table 4 Distribution of risk factors in the MRI in terms of defined thresholds of pathologic values

\begin{tabular}{llll}
\hline & No recurrent dislocation & Recurrent dislocation & $p$ value \\
\hline Age (years) & $18.7 \pm 6.4(16.5-20.8)$ & $18.5 \pm 7.2(15.0-22.0)$ & n.s \\
Female & Male: $22(66.1 \%)$ & Male: $10(52.6 \%)$ & n.s \\
& Female: $14(38.9 \%)$ & Female: $9(47.4 \%)$ & \\
Rupture of the MPFL & $29(80.6 \%)$ & $17(89.5 \%)$ & n.s \\
Postoperative follow-up (years) & $3.9 \pm 1.0(3.5-4.2)$ & $3.7 \pm 1.5(2.8-4.7)$ & \\
Sulcus Angle $\left(^{\circ}\right)$ & $144.1 \pm 5.8(139.9-144.0)$ & $142.0 \pm 6.1(141.3-146.9)$ & n.s \\
Lateral trochlea index $\left(^{\circ}\right)$ & $18.6 \pm 4.9(16.9-20.2)$ & $15.8 \pm 4.7(13.5-18.1)$ & 0.0467 \\
TT-TG (mm) & $13.8 \pm 3.5(12.6-15.0)$ & $15.6 \pm 4.8(13.3-18.0)$ & n.s \\
Patella tilt $\left(^{\circ}\right)$ & $18.9 \pm 5.6(17.0-20.8)$ & $22.6 \pm 8.6(18.5-26.8)$ & n.s \\
Patella shift $(m m)$ & $6.8 \pm 3.5(5.6-8.0)$ & $8.0 \pm 5.7(5.2-10.7)$ & n.s \\
Patellar height & & & \\
Patellotrochlea index $(\%)$ & $0.49 \pm 0.17(0.43-0.54)$ & $0.44 \pm 0.14(0.37-0.51)$ & n.s \\
Koshino index & $1.24 \pm 0.08(1.21-1.26)$ & $1.26 \pm 0.07(1.23-1.30)$ & n.s \\
CDI & $1.22 \pm 0.12(1.18-1.26)$ & $1.25 \pm 0.16(1.18-1.33)$ & n.s \\
BPI & $1.05 \pm 0.13(1.00-1.09)$ & $1.08 \pm 0.17(1.00-1.16)$ & n.s \\
ISI & $1.19 \pm 0.17(1.13-1.25)$ & $1.23 \pm 0.18(1.14-1.32)$ & n.s \\
\hline
\end{tabular}

\begin{tabular}{|c|c|c|c|c|}
\hline & Normal vs. abnormal & $\begin{array}{l}\text { No recurrent } \\
\text { dislocation }\end{array}$ & $\begin{array}{l}\text { Recurrent } \\
\text { dislocation }\end{array}$ & OR $(95 \% \mathrm{CI})$ \\
\hline Age & $\begin{array}{l}\geq 18 \\
<18\end{array}$ & $\begin{array}{l}13(63.9 \%) \\
23(36.1 \%)\end{array}$ & $\begin{array}{l}8(42.1 \%) \\
11(57.89 \%)\end{array}$ & $1.29(0.41-4.01)$ \\
\hline Gender & $\begin{array}{l}\text { Male } \\
\text { Female }\end{array}$ & $\begin{array}{l}22(61.1 \%) \\
14(38.9 \%)\end{array}$ & $\begin{array}{l}10(52.6 \%) \\
9(47.4 \%)\end{array}$ & $1.41(0.46-4.35)$ \\
\hline Sulcus Angle $\left(^{\circ}\right)$ & $\begin{array}{l}\leq 145 \\
>145^{\circ}\end{array}$ & $\begin{array}{l}23(63.9 \%) \\
13(36.1 \%)\end{array}$ & $\begin{array}{l}10(52.6 \%) \\
9(47.4 \%)\end{array}$ & $1.59(0.52-4.92)$ \\
\hline $\begin{array}{l}\text { Lateral trochlea } \\
\text { index }\left({ }^{\circ}\right)\end{array}$ & $\begin{array}{l}\geq 11^{\circ} \\
<11^{\circ}\end{array}$ & $\begin{array}{l}35(97.2 \%) \\
1(2.8 \%)\end{array}$ & $\begin{array}{l}16(84.2 \%) \\
3(15.8 \%)\end{array}$ & $6.56(0.63-68.07)$ \\
\hline TT-TG (mm) & $\begin{array}{l}<16 \mathrm{~mm} \\
\geq 16 \mathrm{~mm}\end{array}$ & $\begin{array}{l}26(72.2 \%) \\
10(27.8 \%)\end{array}$ & $\begin{array}{l}9(47.4 \%) \\
10(52.6 \%)\end{array}$ & $2.89(0.91-9.20)$ \\
\hline Patella tilt $\left(^{\circ}\right)$ & $\begin{array}{l}\leq 20^{\circ} \\
>20^{\circ}\end{array}$ & $\begin{array}{l}21(58.3 \%) \\
15(41.7 \%)\end{array}$ & $\begin{array}{l}8(42.1 \%) \\
11(57.9 \%)\end{array}$ & $1.93(0.62-5.94)$ \\
\hline Patella shift (mm) & $\begin{array}{l}\leq 2.5 \mathrm{~mm} \\
>2.5 \mathrm{~mm}\end{array}$ & $\begin{array}{l}5(13.9 \%) \\
31(86.1 \%)\end{array}$ & $\begin{array}{l}3(15.8 \%) \\
13(84.2 \%)\end{array}$ & $0.86(0.18-4.10)$ \\
\hline \multicolumn{5}{|l|}{ Patellar height } \\
\hline $\begin{array}{l}\text { Patellotrochlea } \\
\text { index }\end{array}$ & $\begin{array}{l}\geq 0.13 \\
<0.13\end{array}$ & $\begin{array}{l}36(100 \%) \\
0\end{array}$ & $\begin{array}{l}19(100 \%) \\
0\end{array}$ & $*$ \\
\hline Koshino index & $\begin{array}{l}\leq 1.2 \\
>1.2\end{array}$ & $\begin{array}{l}12(33.3 \%) \\
24(66.7 \%)\end{array}$ & $\begin{array}{l}5(26.3 \%) \\
14(73.7 \%)\end{array}$ & $1.4(0.41-4.81)$ \\
\hline CDI & $\begin{array}{l}\leq 1.3 \\
>1.3\end{array}$ & $\begin{array}{l}26(72.2 \%) \\
10(27.8 \%)\end{array}$ & $\begin{array}{l}10(52.6 \%) \\
9(47.4 \%)\end{array}$ & $2.34(0.73-7.46)$ \\
\hline BPI & $\begin{array}{l}\leq 1.0 \\
>1.0\end{array}$ & $\begin{array}{l}13(36.1 \%) \\
23(63.9 \%)\end{array}$ & $\begin{array}{l}6(31.6 \%) \\
13(68.4 \%)\end{array}$ & $1.22(0.38-4.00)$ \\
\hline ISI & $\begin{array}{l}\leq 1.3 \\
>1.3\end{array}$ & $\begin{array}{l}26(72.2 \%) \\
10(27.8 \%)\end{array}$ & $\begin{array}{l}10(52.6 \%) \\
9(47.4 \%)\end{array}$ & $2.34(0.73-7.46)$ \\
\hline
\end{tabular}

(*OR could not be calculated) 


\section{Discussion}

The most important finding of the present study was that a ruptured MPFL, an LTI of $<11^{\circ}$, a TT-TG of $\geq 16 \mathrm{~mm}$, and an increased patellar height as per ISI and CDI results in a higher risk $(\mathrm{OR}>2)$ of recurrent patellar dislocation after medial reefing, although the results were not statistically significant. Furthermore, a moderate proportion of patients showed pathological and radiological parameters associated with trochlea dysplasia (sulcus angle $>145^{\circ}: 40 \%$; LTI $\left.<11^{\circ}: 7.2 \%\right)$ and increased patellar height (CDI $>1.3$ : $35 \%$; ISI > 1.3: $35 \%$ ).

Medial reefing has been less evaluated than the MPFL reconstruction or non-surgical treatment of patellar dislocation. In particular, there is no detailed analysis of preoperative MRI parameters that describe indications for medial reefing as well as that evaluate predisposing anatomical factors of recurrent patellar dislocation. Literature describes surgical indications for this procedure mostly based on radiographs. Normal Q-angles and tubercle-sulcus angles as well as no excessive patellar tilt during examination are requirements for medial reefing. A contraindication for medial reefing is a lateral patellofemoral angle on a Merchant radiograph that opens rather medially than laterally [32]. Bodulla et al. [8] described normal bony alignment in addition to the supine $Q$-angles of $<20^{\circ}$, seated tubercle angles of $\leq 5^{\circ}$ and ISI of $<1.2$. Cerciello et al. [12] provided a more comprehensive preoperative assessment of radiological parameters before medial reefing. Imaging, including the weight-bearing anterior-posterior and lateral radiographs of the knee joint, bilateral skyline views at $30^{\circ}$ of flexion, and computed tomography, was performed to assess TT-TG distance and the patellar tilt. MRI is frequently used following patellar dislocation and is important to evaluate anatomical risk factors and concomitant injuries, such as cartilage lesions or osteochondral flakes [43]. This is of particular importance in patients treated with medial reefing because the surgical procedure does not modify these anatomical factors.

Trochlear dysplasia, patellar height, and patellar tilt are known risk factors for primary dislocation. Ridley et al. [34] summarized studies that analyzed anatomical patellofemoral instability imaging measurements in patients with patellofemoral instability and healthy controls. The healthy control group and patients with patellofemoral instability differed with respect to ISI $(1.10$ [95\% CI $1.06-1.15]$ vs. 1.25 [1.22-1.29]), CDI (1.03 [0.93-1.13] vs. $1.24[1.17-1.31])$, patellar tilt $\left(9.19^{\circ}\left[6.58^{\circ}-11.8^{\circ}\right]\right.$ vs. $\left.19.7^{\circ}\left[11.3^{\circ}-28.2^{\circ}\right]\right)$, TT-TG $(9.23[8.22-10.2 \mathrm{~mm}]$ vs. 13.9 [11.6-16.1 $\mathrm{mm}])$, and the sulcus angle $\left(149^{\circ}\right.$ $\left[136^{\circ}-162^{\circ}\right)$ vs. $\left.157^{\circ}\left(152^{\circ}-162^{\circ}\right]\right)$. These patients with a patellar dislocation or patellar instability showed similar values compared with those in the present cohort.

Several authors $[2,16,27]$ described factors associated with a higher risk of recurrent dislocation for different primary treatments, e.g., MPFL reconstructions are more likely to fail in patients with patella alta and an increased TT-TG distance [2, 14, 27]. Arendt et al. [1] analyzed 145 patients and observed that skeletal immaturity, high sulcus angle, and large ISI were significant predictors of recurrent dislocation in patients who underwent non-surgical treatment. Moreover, the cutoff points of a sulcus angle of $\geq 154^{\circ}$ and ISI of $\geq 1.3$ were found. In the presence of these factors, there was a probability of $23 \%$ of recurrent dislocation. Zhang et al. [49] analyzed 166 patients and observed that trochlear dysplasia, elevated TT-TG distance, and patella alta were independently associated with a higher incidence of a second patellar dislocation.

The anatomical risk factors have not been addressed for both non-surgical treatment and medial reefing after primary dislocation. Thus far, there has been no analysis of MRI parameters of patients with medial reefing. Despite the relatively small sample size, similar risk factors for recurrent dislocation compared with non-surgical treatment were found in this study. Similar to Balcarek et al. [3], ORs were calculated to evaluate the influence of MRI parameters on the risk of recurrent dislocation. Balcarek et al. [3] interpreted an OR of $>1.3$ as having a relevant effect on recurrent dislocation, although their results and the results of the present study were not statistically significant. The interpretation of these variables within a surgical algorithm has to be defined [2] and other factors, including activity level, torsional deformities, age, and gender, also need to be considered [26, 29].

The study has several limitations. First, the present study did not include any control or comparison group. It is unclear whether medial reefing is justified for treating primary patellar dislocation, particularly considering the low recurrent dislocation rates of MPFL reconstruction $[9,17$, $24,46]$. Because of the retrospective nature of the present study, selection bias might have occurred leading to an overestimation of the recurrent dislocation rate; it was easier to include patients treated in our clinic owing to recurrent dislocation after medial reefing and to collect MRI data. Moreover, torsional and frontal alignment were not analyzed but are important factors that should be considered [48]. Patients with recurrent dislocation should receive long-leg weight-bearing, and Merchant radiographs, torsional computed tomography, and MRI should be performed to comprehensively assess the anatomical risk factors of patellar dislocation. Moreover, measurements might have been altered, such as the patellar tilt, because of swelling and effusion [3].

Cutoff values for categorizing between the normal and abnormal values were chosen based on literature analysis 
but could have altered statistical findings because the mean differences of MRI parameters between patients with and without recurrent dislocations were small (Table 3). It is difficult to define thresholds because patients without patellar dislocation can have abnormal values, and distinguishing risk factors requires even higher sample sizes. Even in large meta-analysis, the CIs of anatomical risk factors of patients with and without patellar instability are mostly overlapping [34]. Furthermore, differences in these cutoff values can be found frequently between studies, and no consensus has been found yet [5]. In particular, regarding the clinical impact of the cutoff values, further research is needed. In addition, cutoff values are dependent on the image modality [36, 42]. Moreover, the analysis of risk factors is complex because of the interaction of parameters [21]. No statistical corrections for multiple comparisons were calculated. A receiver operating characteristic curve to calculate cohort related cutoff values and multiple regression analysis to distinguish among the influences of different factors could not be performed owing to the relatively small sample size of the present study.

\section{Conclusion}

Anatomical, MRI-based parameters should be considered before indicating medial reefing. A ruptured MPFL, an LTI of $<11^{\circ}$, a TT-TG of $\geq 16 \mathrm{~mm}$, a patellar tilt of $>20 \mathrm{~mm}$, and an increased patellar height according to ISI and CDI were found to be associated, although not significantly, with a higher risk $(\mathrm{OR}>2)$ of recurrent patellar dislocation after medial reefing. Thorough preoperative analysis is crucial to reduce the risk of recurrent dislocation in young patient cohorts.

\footnotetext{
Author's contributions MDA: data analysis, drafting and writing the manuscript, TE: data acquisition, revision of the manuscript. MH: revision of the manuscript. BG: statistical analysis, revision of the manuscript. FS: revision of the manuscript. SD: data acquisition, revision of the manuscript. SS: data acquisition, study design, supervision of the study, revision of the manuscript. CI: data acquisition, writing the manuscript.
}

Funding Open Access funding enabled and organized by Projekt DEAL. No funding was received.

\section{Declarations}

Conflict of interest SS is a member of the AO Joint Preservation and Osteotomy Expert Group. All other authors declare no potential conflict of interest.

Ethical approval The study was approved by the local ethics committee of the University of Tübingen (195/2014BO2).
Informed consent Informed consent was provided by all patients included into the study.

Open Access This article is licensed under a Creative Commons Attribution 4.0 International License, which permits use, sharing, adaptation, distribution and reproduction in any medium or format, as long as you give appropriate credit to the original author(s) and the source, provide a link to the Creative Commons licence, and indicate if changes were made. The images or other third party material in this article are included in the article's Creative Commons licence, unless indicated otherwise in a credit line to the material. If material is not included in the article's Creative Commons licence and your intended use is not permitted by statutory regulation or exceeds the permitted use, you will need to obtain permission directly from the copyright holder. To view a copy of this licence, visit http://creativecommons.org/licenses/by/4.0/.

\section{References}

1. Arendt EA, Askenberger M, Agel J, Tompkins MA (2018) Risk of redislocation after primary patellar dislocation: a clinical prediction model based on magnetic resonance imaging variables. Am J Sports Med 46:3385-3390

2. Arendt EA, England K, Agel J, Tompkins MA (2017) An analysis of knee anatomic imaging factors associated with primary lateral patellar dislocations. Knee Surg Sports Traumatol Arthrosc 25:3099-3107

3. Balcarek P, Oberthür S, Hopfensitz S, Frosch S, Walde TA, Wachowski MM et al (2014) Which patellae are likely to redislocate? Knee Surg Sports Traumatol Arthrosc 22:2308-2314

4. Biedert RM, Albrecht S (2006) The patellotrochlear index: a new index for assessing patellar height. Knee Surg Sports Traumatol Arthrosc 14:707-712

5. Biedert RM, Tscholl PM (2017) Patella Alta: A Comprehensive Review of Current Knowledge. Am J Orthop (Belle Mead NJ) 46:290-300

6. Biesert M, Johansson A, Kostogiannis I, Roberts D (2020) Selfreported and performance-based outcomes following medial patellofemoral ligament reconstruction indicate successful improvements in knee stability after surgery despite remaining limitations in knee function. Knee Surg Sports Traumatol Arthrosc 28:934-940

7. Blackburne JS, Peel TE (1977) A new method of measuring patellar height. J Bone Joint Surg Br 59:241-242

8. Boddula MR, Adamson GJ, Pink MM (2014) Medial reefing without lateral release for recurrent patellar instability: midterm and long-term outcomes. Am J Sports Med 42:216-224

9. Bouras T, Edmond U, Brown A, Gallacher P, Barnett A (2019) Isolated medial patellofemoral ligament reconstruction significantly improved quality of life in patients with recurrent patella dislocation. Knee Surg Sports Traumatol Arthrosc 27:3513-3517

10. Carrillon Y, Abidi H, Dejour D, Fantino O, Moyen B, TranMinh VA (2000) Patellar instability: assessment on MR images by measuring the lateral trochlear inclination-initial experience. Radiology 216:582-585

11. Caton J, Deschamps G, Chambat P, Lerat JL, Dejour H (1982) Patella infera. Apropos of 128 cases. Rev Chir Orthop Reparatrice Appar Mot 68:317-325

12. Cerciello S, Vasso M, Corona K, Del Regno C, Panni AS (2014) Medial capsule reefing in patellar instability. Knee Surg Sports Traumatol Arthrosc 22:2540-2544

13. Dammerer D, Liebensteiner MC, Kujala UM, Emmanuel K, Kopf S, Dirisamer F et al (2018) Validation of the German version 
of the Kujala score in patients with patellofemoral instability: a prospective multi-centre study. Arch Orthop Trauma Surg 138:527-535

14. Dejour H, Walch G, Nove-Josserand L, Guier C (1994) Factors of patellar instability: an anatomic radiographic study. Knee Surg Sports Traumatol Arthrosc 2:19-26

15. Erickson BJ, Mascarenhas R, Sayegh ET, Saltzman B, Verma NN, Bush-Joseph CA et al (2015) Does operative treatment of first-time patellar dislocations lead to increased patellofemoral stability? A systematic review of overlapping meta-analyses. Arthroscopy 31:1207-1215

16. Feucht MJ, Mehl J, Forkel P, Achtnich A, Schmitt A, Izadpanah $\mathrm{K}$ et al (2020) Failure analysis in patients with patellar redislocation after primary isolated medial patellofemoral ligament reconstruction. Orthop J Sports Med 8:2325967120926178

17. Flanigan DC, Shemory S, Lundy N, Stitgen M, Long JM, Magnussen RA (2020) Medial patellofemoral ligament reconstruction with allograft versus autograft tissue results in similar recurrent dislocation risk and patient-reported outcomes. Knee Surg Sports Traumatol Arthrosc 28:2099-2104

18. Frings J, Balcarek P, Tscholl P, Liebensteiner M, Dirisamer F, Koenen P (2020) Conservative versus surgical treatment for primary patellar dislocation. Dtsch Arztebl Int 117:279-286

19. Gravesen KS, Kallemose T, Blond L, Troelsen A, Barfod KW (2018) High incidence of acute and recurrent patellar dislocations: a retrospective nationwide epidemiological study involving 24.154 primary dislocations. Knee Surg Sports Traumatol Arthrosc 26:1204-1209

20. Ihle C, Maurer J, Ziegler P, Stockle U, Ateschrang A, Ahrend MD et al (2019) Sporting activity is reduced following medial reefing performed for patellar dislocation: a retrospective case series of 144 patients with a minimum follow-up of 24 months. BMC Musculoskelet Disord 20:34

21. Imhoff FB, Funke V, Muench LN, Sauter A, Englmaier M, Woertler K et al (2020) The complexity of bony malalignment in patellofemoral disorders: femoral and tibial torsion, trochlear dysplasia, TT-TG distance, and frontal mechanical axis correlate with each other. Knee Surg Sports Traumatol Arthrosc 28:897-904

22. Insall J, Salvati E (1971) Patella position in the normal knee joint. Radiology 101:101-104

23. Irrgang JJ, Anderson AF, Boland AL, Harner $\mathrm{CD}$, Kurosaka M, Neyret $P$ et al (2001) Development and validation of the international knee documentation committee subjective knee form. Am J Sports Med 29:600-613

24. Kang H, Zheng R, Dai Y, Lu J, Wang F (2019) Single- and double-bundle medial patellofemoral ligament reconstruction procedures result in similar recurrent dislocation rates and improvements in knee function: a systematic review. Knee Surg Sports Traumatol Arthrosc 27:827-836

25. Keser S, Savranlar A, Bayar A, Ege A, Turhan E (2008) Is there a relationship between anterior knee pain and femoral trochlear dysplasia? Assessment of lateral trochlear inclination by magnetic resonance imaging. Knee Surg Sports Traumatol Arthrosc 16:911

26. Koh Y-G, Nam J-H, Chung H-S, Lee H-Y, Kim H-J, Kim H-J et al (2019) Gender-related morphological differences in sulcus angle and condylar height for the femoral trochlea using magnetic resonance imaging. Knee Surg Sports Traumatol Arthrosc $27: 3560-3566$

27. Köhlitz T, Scheffler S, Jung T, Hoburg A, Vollnberg B, Wiener E et al (2013) Prevalence and patterns of anatomical risk factors in patients after patellar dislocation: a case control study using MRI. Eur Radiol 23:1067-1074
28. Koshino T, Sugimoto K (1989) New measurement of patellar height in the knees of children using the epiphyseal line midpoint. J Pediatr Orthop 9:216-218

29. Lin KM, James EW, Aitchison AH, Schlichte LM, Wang G, Green DW (2021) Increased tibiofemoral rotation on MRI with increasing clinical severity of patellar instability. Knee Surg Sports Traumatol Arthrosc. https://doi.org/10.1007/ s00167-020-06382-x

30. Marcheggiani Muccioli GM, Lullini G, Grassi A, Macchiarola L, Cammisa E, Maccaferri B et al (2021) Good results are reported at 60 -month follow-up after medial patello-femoral ligament reconstruction with fascia lata allograft for recurrent patellar dislocation. Knee Surg Sports Traumatol Arthrosc 29:1191-1196

31. Mehl J, Otto A, Comer B, Kia C, Liska F, Obopilwe E et al (2020) Repair of the medial patellofemoral ligament with suture tape augmentation leads to similar primary contact pressures and joint kinematics like reconstruction with a tendon graft: a biomechanical comparison. Knee Surg Sports Traumatol Arthrosc 28:478-488

32. Miller JR, Adamson GJ, Pink MM, Fraipont MJ, Durand P Jr (2007) Arthroscopically assisted medial reefing without routine lateral release for patellar instability. Am J Sports Med 35:622-629

33. Muellner T, Funovics M, Nikolic A, Metz V, Schabus R, Vecsei V (1998) Patellar alignment evaluated by MRI. Acta Orthop Scand 69:489-492

34. Ridley TJ, Hinckel BB, Kruckeberg BM, Agel J, Arendt EA (2016) Anatomical patella instability risk factors on MRI show sensitivity without specificity in patients with patellofemoral instability: a systematic review. J ISAKOS 1:141-152

35. Saccomanno MF, Sircana G, Fodale M, Donati F, Milano G (2016) Surgical versus conservative treatment of primary patellar dislocation. A systematic review and meta-analysis. Int Orthop 40:2277-2287

36. Salzmann GM, Weber TS, Spang JT, Imhoff AB, Schottle PB (2010) Comparison of native axial radiographs with axial MR imaging for determination of the trochlear morphology in patients with trochlear dysplasia. Arch Orthop Trauma Surg 130:335-340

37. Schmeling A. Aktuelle Aspekte der patellofemoralen Instabilität (2010). https://www.dgou.de/fileadmin/user_upload/Dokum ente/Publikationen/SFA/SFA_Aktuell_Nr23.pdf. Accessed 06 June 2020

38. Schoettle PB, Zanetti M, Seifert B, Pfirrmann CW, Fucentese SF, Romero J (2006) The tibial tuberosity-trochlear groove distance; a comparative study between CT and MRI scanning. Knee 13:26-31

39. Shrout PE, Fleiss JL (1979) Intraclass correlations: uses in assessing rater reliability. Psychol Bull 86:420

40. Smith TO, Donell S, Song F, Hing CB (2015) Surgical versus non-surgical interventions for treating patellar dislocation. Cochrane Database Syst Rev (2). https://doi.org/10.1002/14651 858.CD008106.pub3CD008106

41. Uimonen MM, Repo JP, Huttunen TT, Nurmi H, Mattila VM, Paloneva J (2020) Surgery for patellar dislocation has evolved towards anatomical reconstructions with assessment and treatment of anatomical risk factors. Knee Surg Sports Traumatol Arthrosc. https://doi.org/10.1007/s00167-020-06277-x

42. Verhulst FV, van Sambeeck JD, Olthuis GS, van der Ree J, Koëter S (2020) Patellar height measurements: Insall-Salvati ratio is most reliable method. Knee Surg Sports Traumatol Arthrosc 28:869-875

43. Vollnberg B, Koehlitz T, Jung T, Scheffler S, Hoburg A, Khandker D et al (2012) Prevalence of cartilage lesions and early 
osteoarthritis in patients with patellar dislocation. Eur Radiol 22:2347-2356

44. Wang SN, Qin CH, Jiang N, Wang BW, Wang L, Yu B (2016) Is surgical treatment better than conservative treatment for primary patellar dislocations? A meta-analysis of randomized controlled trials. Arch Orthop Trauma Surg 136:371-379

45. Waterman BR, Belmont PJ Jr, Owens BD (2012) Patellar dislocation in the United States: role of sex, age, race, and athletic participation. J Knee Surg 25:51-57

46. Wilkens OE, Hannink G, van de Groes SAW (2020) Recurrent patellofemoral instability rates after MPFL reconstruction techniques are in the range of instability rates after other soft tissue realignment techniques. Knee Surg Sports Traumatol Arthrosc 28:1919-1931

47. Wong TT, Denning J, Moy MP, Rasiej MJ, Redler LH, Ahmad CS et al (2021) MRI following medial patellofemoral ligament reconstruction: assessment of imaging features found with post-operative pain, arthritis, and graft failure. Skeletal radiol 50:981-991

48. Xu Z, Zhang H, Guo M, Wen Z, Zhang J, Zhou A (2021) Malalignment sign on knee magnetic resonance imaging: a new predictor for excessive femoral anteversion in patients with patellar dislocation. Knee Surg Sports Traumatol Arthrosc 29:1075-1082

49. Zhang G-y, Ding H-y, Li E-m, Zheng L, Bai Z-w, Shi H et al (2019) Incidence of second-time lateral patellar dislocation is associated with anatomic factors, age and injury patterns of medial patellofemoral ligament in first-time lateral patellar dislocation: a prospective magnetic resonance imaging study with 5-year follow-up. Knee Surg Sports Traumatol Arthrosc $27: 197-205$

Publisher's Note Springer Nature remains neutral with regard to jurisdictional claims in published maps and institutional affiliations. 